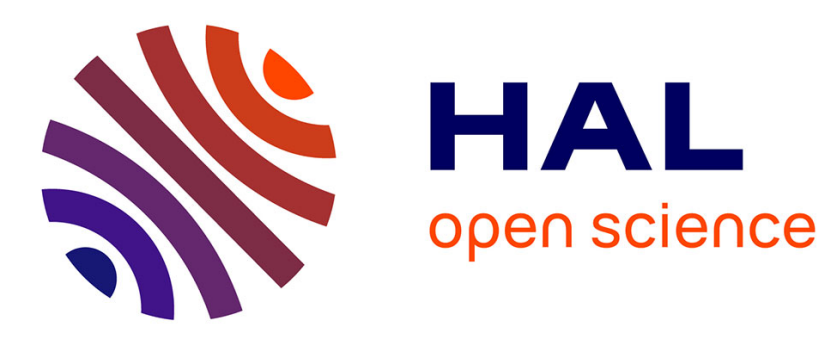

\title{
Real-Time Study of Coexisting States in Laser Cavity Solitons
}

Pierre Henry Hanzard, Maxwell Rowley, Antonio Cutrona, Sai Chu, Brent

Little, Roberto Morandotti, David Moss, Benjamin Wetzel, Juan Sebastian Totero Gongora, Marco Peccianti, et al.

\section{To cite this version:}

Pierre Henry Hanzard, Maxwell Rowley, Antonio Cutrona, Sai Chu, Brent Little, et al.. Real-Time Study of Coexisting States in Laser Cavity Solitons. CLEO: QELS_Fundamental Science, 2021, San Jose, United States. pp.FM4H.7, 10.1364/CLEO_QELS.2021.FM4H.7 . hal-03417992

\section{HAL Id: hal-03417992 \\ https://hal.science/hal-03417992}

Submitted on 19 Nov 2021

HAL is a multi-disciplinary open access archive for the deposit and dissemination of scientific research documents, whether they are published or not. The documents may come from teaching and research institutions in France or abroad, or from public or private research centers.
L'archive ouverte pluridisciplinaire HAL, est destinée au dépôt et à la diffusion de documents scientifiques de niveau recherche, publiés ou non, émanant des établissements d'enseignement et de recherche français ou étrangers, des laboratoires publics ou privés. 


\title{
Real-Time Study of Coexisting States in Laser Cavity Solitons
}

\author{
Pierre Henry Hanzard ${ }^{1 *}$, Maxwell Rowley ${ }^{1}$, Antonio Cutrona ${ }^{1}$, Sai T. Chu ${ }^{2}$, Brent E. Little ${ }^{3}$, Roberto \\ Morandotti $^{4,5}$, David J. Moss ${ }^{6}$, Benjamin Wetzel ${ }^{7}$, Juan Sebastian Totero Gongora ${ }^{1}$, Marco Peccianti ${ }^{1}$ and \\ Alessia Pasquazi ${ }^{1}$ \\ ${ }^{1}$ Emergent Photonics (Epic) Lab, Dept. of Physics and Astronomy, University of Sussex, BN1 9QH, UK \\ ${ }^{2}$ Department of Physics, City University of Hong Kong, Tat Chee Avenue, Hong Kong, China SAR \\ ${ }^{3}$ State Key Laboratory of Transient Optics and Photonics, Xi'an Institute of Optics and Precision Mechanics, CAS, Xi'an, China \\ ${ }^{4}$ INRS-EMT, 1650 Boulevard Lionel-Boulet, Varennes, Québec, Canada J3X 1S2 \\ ${ }^{5}$ Institute of Fundamental and Frontier Sciences, University of Electronic Science and Technology, Chengdu, China \\ ${ }^{6}$ Optical Sciences Centre, Swinburne University of Technology, Hawthorn, VIC 3122, Australia \\ ${ }^{7}$ Xlim Research Institute, CNRS UMR 7252, Université de Limoges, 87060 Limoges, France \\ *p.b.hanzard@sussex.ac.uk
}

\begin{abstract}
We experimentally demonstrate the presence of two coexisting states in Laser Cavity Solitons (LCS) Microcombs. By using the Dispersive Fourier Transform technique, we show the simultaneous presence of both LCS and a background modulation. (C) 2021 The Author(s)
\end{abstract}

\section{Introduction}

Laser cavity-solitons microcombs have recently been introduced by our team at the University of Sussex [1]. In such microcombs, self-localized waves have shown generation of a broad-spectrum with high average powers and an unparalleled mode efficiency, compared to traditional micro-cavity-based frequency combs. A typical implementation of the setup able to produce this new category of waves is depicted in Fig. 1(a) and includes a nonlinear Kerr micro-cavity - responsible for the broadband generation - nested in a fibre amplifying loop, which provides energy to the system. Very importantly, LCS microcombs appear to be robust against perturbations and controllable with simple parameters, such as cavity length and amplifier gain current, in stark contrast with today's state-of-the-art. To gain insight into the dynamics, however, they need to be studied with an extremely high temporal resolution, as they are ultrashort pulses of hundreds of femtoseconds. At the same time, on a long temporal span, to observe their formation and interaction, usually mediated by thermal and gain dynamics acting in the micro-to-millisecond time scales. These challenging temporal features require the development of ad-hoc metrological tools. Recently, powerful real-time characterisation techniques for ultrashort pulses have emerged in photonics. The capacity of these tools to record ultrafast events (below picosecond timescale), while being able to measure, at the same time, long temporal spans (few milliseconds) has opened the research field of so-called 'non-repetitive' events [2]. In nonlinear optics, this includes the study of optical rogue waves [3] and solitons collision-induced explosion in mode-locked lasers [4]. These techniques also permitted to deeply understand ultrafast laser start-up regimes, by observing the build-up of femtosecond mode-locking, from noise through Qswitching to a stable mode-locking regime [5].

\section{Experimental results and discussions}

The Dispersive Fourier Transform (DFT) technique is a powerful tool for characterizing the spectrum of optical pulses in real-time. However, this technique relies on temporally stretching optical pulses in a fibre, which can result in pulse overlapping and thus information losses. Our setup delivers pulses at a very high repetition rate $(50 \mathrm{GHz})$, leaving very little space for the pulses to stretch and reaching the so-called far-field condition, at which the temporal profile of the pulse has the shape of its spectrum. This problem is solved by optically sampling our laser emission rate at a reduced repetition rate using amplitude modulation (Fig. 1(b)). By lowering the repetition rate to $33 \mathrm{MHz}$, we manage to reduce the signal duty cycle thus allowing pulses temporal stretching without overlap. Nevertheless, as our modulation system speed does nott allow for selecting single pulses, bunches of 10 pulses are kept and the approximation that these can be considered as one average pulse is made. We justify this approximation by the fact that this study is focused over longer timescales for which the pulse-to-pulse variation is negligible. A very interesting feature within our DFT implementation is the ability to observe a signature of the radio-frequency response of the laser directly superimposed to the DFT trace. This allows us to study a set of peculiar laser states composed by the superposition of a Laser Cavity Soliton with a continuous wave/Turing pattern state. Solitons and Turing patterns in our system are characterised by the locking of the laser lines on the 
red/ blue detuned slope of the microcavity, respectively. Differently from externally pumped microcavities, our system allows the coexistence of these states. Such configuration can be achieved when the mode spacing (freespectral range) of the fibre laser cavity is within the order of the microcavity linewidth. In this case, a blue and a red detuned line are allowed to oscillate simultaneously in the microcavity resonance, and such different nonlinear states can coexist. Their signature is the presence of a radiofrequency beating approximately equal to the free spectral range of the amplifier cavity.

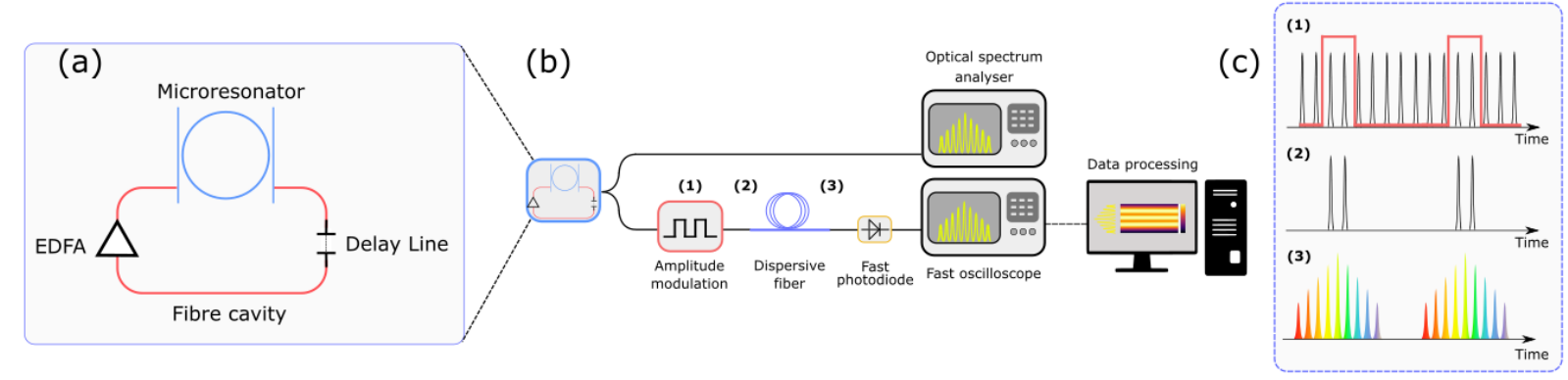

Figure 1. (a) LCS are generated using a micro-resonator nested in a fibre cavity. (b) Real-time diagnostic setup. Amplitude modulation is first used to reduce LCS repetition rate, allowing them to temporally stretch in a dispersion compensation

fibre. The pulses are then recorded using a fast detection setup comprising a fast photodetector and oscilloscope.

Here we present an experimental study that shows the dynamical evolution of the two superimposed states. A typical DFT measurement demonstrating the coexistence of a LCS with a continuous wave state is reported (Fig. 2). In particular, we observe the temporal buildup of the LCS, with the DFT showing the formation of the comblike optical spectrum visible after $20 \mu \mathrm{s}$ - and centred at $1540 \mathrm{~nm}$. Superimposed to this spectrum, it is possible to observe a slow modulation with a frequency of approximately $80 \mathrm{MHz}$, matching the free-spectral range of our laser cavity. This modulation is due to the presence of a continuous wave state at $1540 \mathrm{~nm}$ which appears simultaneously with the pulsed regime. The evolution of the modulation in time indicates that the relative position of the laser lines within the microcavity is changing. In particular, it indicates that the two states are getting less frequency detuned until $150 \mu \mathrm{s}$, and the detuning then increases after that point, eventually stabilising to around $80 \mathrm{MHz}$. The coexistence of these two states, once a stationary regime is reached, can be confirmed by a laserscanning spectroscopy measurement. We will present a detailed discussion of our DFT technique for the study of LCS.

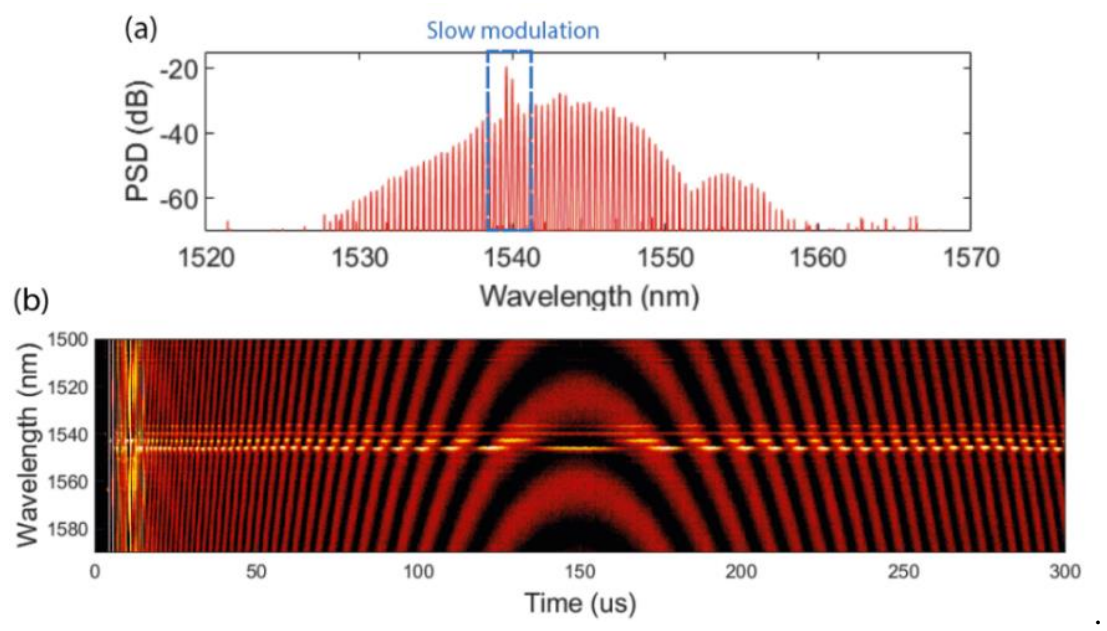

Figure 2 (a) Optical spectrum of the LCS. The spectral lines responsible for the slow modulation are circled in blue. (b) Start-up of the LCS regime $(0-20 \mu \mathrm{s})$. The radio-frequency beating can be observed over hundreds of microseconds.

\section{References}

[1] Bao, H, et al. Nat. Photonics (2019). doi:10.1038/s41566-019-03795

[2] K. Goda , et al. Nature Photonics, p. 102, 2013

[3] Solli, D. R, et al. Nature 450.7172 (2007): 1054

[4] Peng, J, et al. Commun. Phys. 2, (2019)

[5] Herink, G, et al. Nat. Photonics 10, 321-326 (2016) 